\title{
Basic Elements Knowledge Acquisition Study in the Chinese Character Intelligent Formation System
}

\author{
Mingyou LIU, Chengsen DUAN, Youguo PI \\ South China University of Technology, Guangzhou, China. \\ Email: liumingyou1025@gmail.com \\ Received July $27^{\text {th }}, 2009$; revised August $22^{\text {nd }}, 2009$; accepted September $7^{\text {th }}, 2009$.
}

\begin{abstract}
In the Chinese character intelligent formation system without Chinese character library, it is possible that the same basic element in different Chinese characters is different in position, size and shape. The geometry transformation from basic elements to the components of Chinese characters can be realized by affine transformation, the transformation knowledge acquisition is the premise of Chinese character intelligent formation. A novel algorithm is proposed to acquire the affine transformation knowledge of basic elements automatically in this paper. The interested region of Chinese character image is determined by the structure of the Chinese character. Scale invariant and location invariant of basic element and Chinese character image are extracted with SIFT features, the matching points of the two images are determined according to the principle of Minimum Euclidean distance of eigenvectors. Using corner points as identification features, calculating the one-way Hausdorff distance between corner points as the similarity measurement from the affine image to the Chinese character sub-image, affine coefficients are determined by optimal similarity. 70244 Chinese characters in National Standards GB18030-2005 character set are taken as the experimental object, all the characters are performed and the experimental courses and results are presented in this paper.
\end{abstract}

Keywords: Chinese Character Intelligent Formation, Knowledge Acquisition, Affine Transformation, Hausdorff Distance

\section{Introduction}

The concept of Chinese character intelligent information that reuse basic elements to form Chinese characters was proposed based on prototype authentication mechanism of cognitive psychology in the reference [1], the author identified that replace the Chinese character library by Chinese character intelligent information. In his further research, the framework of the Chinese character intelligent formation system which contains basic elements library, knowledge library and Chinese character intelligent formation model [2] was proposed, and the structure knowledge of the Chinese character is obtained by simple grid [3-5].

The spelling of alphabetic character has a one-dimensional characteristic; it is pieced together in order under a few rules. But the Chinese character has the two-dimensional characteristic, the same basic element in different Chinese characters is possible different in its size, shape and position. The mapping from basic elements to the components of the Chinese character can be realized by affine transformation. A method that reuses radicals to generate Chinese characters based on global affine transformation was proposed in the reference [6]; the affine coefficients were calculated by minimizing the mean of the nearest-neighbor inter-point distance. But this method was complex and it is easy to bring errors resulted from image processing, such as outline extraction. A novel approach was proposed that selects corresponding points in the boundary box of the basic elements and mapping goal respectively to calculate the affine coefficients, then optimized by PSO algorithm, but this method need to remove other components manually when get the bounding box of mapping goal, so the workload was heavy.

In this paper, the solution for acquiring basic elements' affine coefficients is as follows, the interested region of Chinese character image is determined by the structure of the Chinese character. Scale invariant and location invariant of basic element and Chinese character image are extracted with SIFT features, matching points of the two images are determined according to the principle of Euclidean distance of eigenvectors. One group of affine coefficients can be calculated from three pairs of match- 
ing points which are chosen randomly. The affine image is obtained through the transformation from the basic element image, the corner points of affine image and Chinese character sub-image are detected, one-way Hausdorff distance between corner points is used as the rule of measuring the similarity, and the optimal affine coefficients are determined by minimum one-way Hausdorff distance.

The contents of this paper is arranged as follows, the principle of Chinese character intelligent information is presented in Section 2; the geometric transformation model of the basic elements are proposed in Section 3, the size, position and shape changes of basic elements can be realized by affine transformation; in Section 4, a new method of acquiring the affine coefficients of the basic elements is proposed; the experiment study is presented in Section 5, the generation of more than 70000 Chinese characters is carried out after acquired the transformation knowledge in different Chinese characters of all basic elements. Finally, the conclusions are given in Section 6.

\section{Theory of the Chinese Character Intelligent Formation}

A Chinese character is a combination of either single hieroglyphic or self-explanatory symbol, or several of them based on meaning and echoism rules. Any element in the set of the character components corresponds to a certain basic element. The components of Chinese characters are the topological mapping of basic elements in the character structure, namely, the position, size and shape of basic elements may be different in different Chinese characters, but basic elements are topological invariant. For example, basic elements “y” and “少” have different size, shape and position in the characters “沙” and “莎”. As another example of this, basic element “才” has different size, shape and position in the characters “讨”, “辱” and “裖”. In the above-mentioned transformation, the components which are corresponding to the same basic element in different Chinese characters are different in size, shape and position, but they have the same topological structure, namely, they have topological invariance.

From the above analysis, the concept of Chinese character intelligent information was proposed. Basic elements include single hieroglyphic and self-explanatory and their symbols; a Chinese character is generated by putting one or more basic elements together after topological mapping to the structure of the Chinese character. Take the generation of the Chinese character “磨”" as an example, as shown in Figure 1. In order to facilitate understanding, the decomposition of structures and basic elements of the character is illustrated in Figure 2. This character contains four level structure and five components mapped by four basic elements. The symbols "\#", “木”, “广”, “石” are basic elements, $F_{i}(i=1,2,3,4,5)$ represents the knowledge of the topological mappings, such as the location, size and shape of basic elements in the Chinese character. The final Chinese character “蘑” is formed by mapping the basic elements to the corresponding structure of the Chinese character.

\section{Transformation Model of Basic Elements}

From the analysis in Section 2, it needs a topological transformation method which can realize to transform basic elements to different position, size and shape in order to realize the mapping from basic elements to components of the Chinese character, affine transformation meets this requirement. Suppose $W$ is a basic elements image and $x$ is a point of the image. Let us define a geometric transformation of the basic element image by

$$
A W+t=\left(\begin{array}{ll}
a_{A} & b_{A} \\
c_{A} & d_{A}
\end{array}\right) W+t=\left(\begin{array}{ll}
a_{A} & b_{A} \\
c_{A} & d_{A}
\end{array}\right)\left(\begin{array}{l}
W_{x} \\
W_{y}
\end{array}\right)+\left(\begin{array}{l}
t_{x} \\
t_{y}
\end{array}\right)
$$

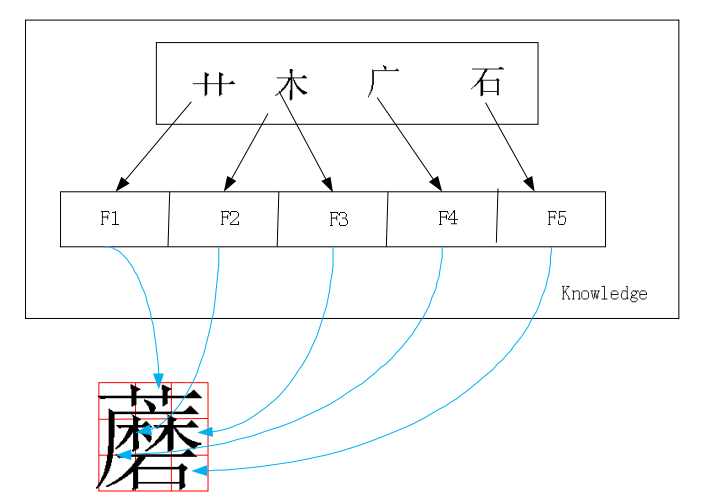

Figure 1. The theory of Chinese character intelligent formation

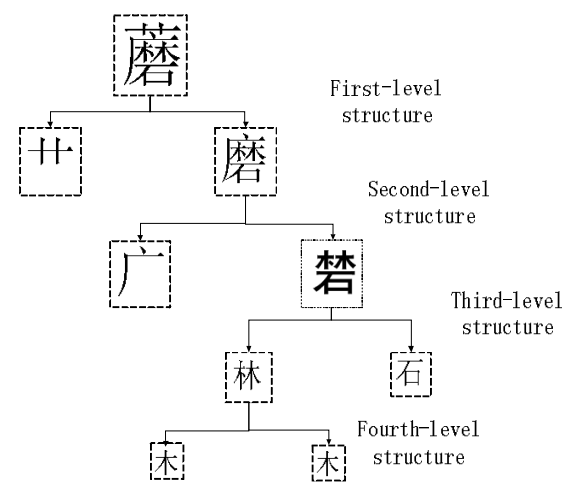

Figure 2. Example of the decomposition of structures and basic elements 
With $A$ corresponding to a linear transformation and $t$ a translation vector. Of which, $a_{A}$ and $d_{A}, b_{A}$ and $c_{A}, t_{x}$ and $t_{y}$ represent scaling factor, rotation factor and translation along the $x, y$ axes respectively. The Chinese characters are standard-type-face characters that square in a box, which determined the geometric transformation of basic elements involves only non-uniform scale, without rotation, that is, $b_{A}=c_{A}=0$.

\section{Knowledge Acquisition of Basic Elements}

The flow of acquiring the affine coefficients of basic elements that proposed in this paper is as follows. Firstly, the interested region of character image is determined by the structure of the Chinese character. Secondly, the scale and location invariant of basic element and Chinese character image are extracted by SIFT algorithm, and the matching points of two images are determined by nearest Euclidean distance of eigenvectors. Of all the key matching points, a few are incorrect, it is not necessary to remove all the pairs of mismatched points, because it can calculate affine coefficients with three pairs of correct matching points. Thirdly, three pairs of non-collinear points are chosen randomly to calculate one group of affine coefficients, the basic element image is transformed to obtain affine image, the corner points of affine and Chinese character sub-image are detected respectively, and the one-way Hausdorff distance of corner points from affine image to Chinese character sub-image is calculated. Finally, the optimal affine coefficients are determined according to the minimum one-way Hausdorff distance after limited iterations.

\subsection{Region of Interest}

The combinational relation of location among the components of the Chinese character is considered as the structure of the Chinese character. The location of different components in a Chinese character is determined, thus, a rectangular region can be fixed by the structure of Chinese character, called region of interest, which only contains one of the components of the Chinese character.

Through the analysis of the components' combinational relations of the Chinese character, the structure types of the Chinese character are summarized. The structure of the Chinese character can divided into several levels, which depend on whether there is combination of basic elements, the structure without combination of basic elements is called one-level structure, while multiple-level structure is with a combination of basic elements. The structure level of the Chinese characters obeys the cognitive mechanism that is from left to right, from top to bottom and from out to inner, for example, the character “蘑” contains four level structures, as shown in Figure 2.

Suppose the region of the Chinese character is $T(x, y)$ :

$$
T(x, y)=\left\{\begin{array}{l}
0 \leq x \leq w \\
0 \leq y \leq h
\end{array}\right.
$$

Where $w$ and $h$ are the width and height of the Chinese character image.

The code structure of the Chinese character is a kind of hierarchical level, along the level-dividing tree, the interested region of secondary level structure is contained in its interested region of previous level structure. Suppose the interested region of the previous level structure and its sub-level structure can be represented with $R(x, y)$ and $S(x, y)$ respectively as follows.

$$
\begin{aligned}
& R(x, y)= \begin{cases}r_{x 1} \leq x \leq r_{x 2} & \left(0 \leq r_{x 1} \leq r_{x 2} \leq w\right) \\
r_{y 1} \leq y \leq r_{y 2} & \left(0 \leq r_{y 1} \leq r_{y 2} \leq h\right)\end{cases} \\
& S(x, y)= \begin{cases}s_{x 1} \leq x \leq s_{x 2} & \left(0 \leq s_{x 1} \leq s_{x 2} \leq w\right) \\
s_{y 1} \leq y \leq s_{y 2} & \left(0 \leq s_{y 1} \leq s_{y 2} \leq h\right)\end{cases}
\end{aligned}
$$

From the above analysis, it can be obtained:

$$
\begin{aligned}
& S(x, y) \subseteq R(x, y), \quad 0 \leq r_{x 1} \leq s_{x 1} \leq s_{x 2} \leq r_{x 2} \leq w, \\
& 0 \leq r_{y 1} \leq s_{y 1} \leq s_{y 2} \leq r_{y 2} \leq h .
\end{aligned}
$$

In order to calculate the interested region of each basic element conveniently, the relation between the interested region of the previous level structure and its sub-level structure can be defined as follows.

$$
\mathrm{h}(a, b)= \begin{cases}\alpha_{1} \leq a \leq \alpha_{2} & \left(0 \leq \alpha_{1} \leq \alpha_{1} \leq 1\right) \\ \beta_{1} \leq b \leq \beta_{2} & \left(0 \leq \beta_{1} \leq \beta_{2} \leq 1\right)\end{cases}
$$

Where $\alpha_{1}$ and $\alpha_{2}, \beta_{1}$ and $\beta_{2}$ represent the percentages of the interested region of the sub-level structure holding that of it's previous level structure in $\mathrm{X}$ and $\mathrm{Y}$ axis respectively, which can be set by actual situation. Through the analysis above, the interested region of sub-structure comparing with its previous structure can be calculated as follows.

$$
S(x, y)=\left\{\begin{array}{l}
r_{x 1}+\alpha_{1} \times\left(r_{x 2}-r_{x 1}\right) \leq x \leq r_{x 1}+\alpha_{2} \times\left(r_{x 2}-r_{x 1}\right) \\
r_{y 1}+\beta_{1} \times\left(r_{y 2}-r_{y 1}\right) \leq y \leq r_{y 1}+\beta_{2} \times\left(r_{y 2}-r_{y 1}\right)
\end{array}\right.
$$

The interested region of each basic element in the Chinese character can be calculated along the level-dividing tree of Chinese characters' code structure with the method of recursive under Equation (6).

\subsection{Extract Scale and Location Invariant with SIFT}

The SIFT $[7,8]$ features are local and based on the appearance of the object at particular interest points, and are invariant to image scale and rotation.

According the characteristic of the Chinese character, this article improved the SIFT that remove the rotation invariant and extract scale and location invariant of SIFT features. The main steps are included as follows. 
For this, the image is convolved with Gaussian filters at different scales, and then the difference of successive Gaussian-blurred images are taken, Key points are then taken as maxima/minima of the Difference of Gaussians that occur at multiple scales. Specifically, a DoG image $G(x, y, \sigma)$ is given by

$$
\begin{aligned}
& D(x, y, \sigma)=(G(x, y, k \sigma)-G(x, y, \sigma)) * I(x, y) \\
& =L(x, y, k \sigma)-L(x, y, \sigma)
\end{aligned}
$$

Where $L(x, y, k \sigma)$ is the original image $I(x, y)$ convolved with the Gaussian blur $G(x, y, k \sigma)$ at scale $k \sigma$

$$
L(x, y, \sigma)=G(x, y \sigma) * I(x, y)
$$

This is done by comparing each pixel in the DoG images to its eight neighbors at the same scale and nine corresponding neighboring pixels in each of the neighboring scales. If the pixel value is the maximum or minimum among all compared pixels, it is selected as a candidate key point.

Scale-space extreme detection produces too many key point candidates, some of which are unstable. The next step in the algorithm is to perform a detailed fit to the nearby data for accurate location, scale, and ratio of principal curvatures. This information allows points to be rejected that have low contrast (and are therefore sensitive to noise) or are poorly localized along an edge.

The Gaussian-smoothed image $L(x, y, \sigma)$ at the key point's scale $\sigma$ is taken so that all computations are performed in a scale-invariant manner. For an image sample $L(x, y)$ at scale $\sigma$, the gradient magnitude, $m(x, y)$, and orientation, $\theta(x, y)$, are computed using pixel differences:

$$
\begin{aligned}
& m(x, y)= \\
& \sqrt{(L(x+1, y)-L(x-1, y))^{2}+(L(x, y+1)-L(x, y-1))^{2}}
\end{aligned}
$$

$$
\begin{aligned}
& \theta(x, y)=\arctan ((L(x, y+1)-L(x, y-1)) \\
& /(L(x+1, y)-L(x-1, y)))
\end{aligned}
$$

Previous steps found key point locations at particular scales and assigned orientations to them. This ensured invariance to image location, scale. Now we want to compute descriptor vectors for these key points such that the descriptors are highly distinctive and partially invariant to the remaining variations, like illumination, 3D viewpoint, etc. This step is pretty similar to the Orientation Assignment step. The feature descriptor is computed as a set of orientation histograms on (4 $x$ 4) pixel neighborhoods. Just like before, the contribution of each pixel is weighted by the gradient magnitude, and by a Gaussian with $\sigma 1.5$ times the scale of the key point. Histograms contain 8 bins each, and each descriptor contains a $4 \times 4$ array of 16 histograms around the key point. This leads to a SIFT feature vector with $(4 \times 4 \times 8$ $=128$ elements). This vector is normalized to enhance invariance to changes in illumination.

\subsection{Key Points Match}

When the key point descriptor of two images was built, take the Euclidean distance between the descriptors of each feature point as the measurement of the similarity. Suppose the sets of descriptor are, $F_{a}=\left\{f_{1}^{(a)}, f_{2}^{(a)}, \mathrm{L} f_{N a}^{(a)}\right\}$, $F_{b}=\left\{f_{1}^{(b)}, f_{2}^{(b)}, \mathrm{L} f_{N b}^{(b)}\right\}$ respectively in images $\mathrm{A}$ and $\mathrm{B}$, where $\mathrm{Na}$ and $\mathrm{Nb}$ are the numbers of key points in the two images respectively, the Euclidean distance $d$ between any descriptor $f_{i}^{(a)}$ in image A to any descriptor $f_{j}^{(b)}$ in image $\mathrm{B}$ is presented as below,

$$
d\left(f_{i}^{(a)}, f_{j}^{(b)}\right)=\sqrt{\sum_{d=0}^{D-1}\left(f_{i}^{(a)}[d]-f_{j}^{(b)}[d]\right)^{2}}
$$

Take one of the key points in image A, and find the key point with nearest Euclidean distance in image B. In the case of the ratio of nearest Euclidean distance and the second nearest Euclidean distance, it is hard to choose a threshold, if the threshold value is too small, the correct matching points pairs may get lost. So, this method avoids losing correct matching points pairs.

\subsection{Hausdorff Distance}

In this paper, the corner points is taken as the recognition clue, the similarity measurement between affine and character sub-image is realized by using one-way Hausdorff distance. Firstly, the boundary box of affine image is obtained, a region in the character sub-image is determined corresponding to the boundary box and is extracted. Secondly, the corner points are detected in affine image and character sub-image. Common corner points detection algorithm are mainly Harris, Susan, etc, this paper adopts the algorithm that proposed by Shi.J and Tomasi.C [9].

Assumed two finite sets $S=\left\{s_{1}, s_{2}, \ldots, s_{p}\right\}$ and $T=\left\{t_{1}\right.$, $\left.\mathrm{t}_{2}, \ldots, \mathrm{t}_{\mathrm{q}}\right\}$, the Hausdorff distance [10] is given as,

$$
\mathrm{H}(\mathrm{S}, T)=\max (\mathrm{h}(S, T), h(T, S))
$$

Where $h(S, T)=\max _{s \in S} \min _{t \in T}\|s-t\|$,

$$
h(T, S)=\max _{t \in T} \min _{s \in S}\|t-s\|
$$

Where $\|\bullet\|$ represents a distance norm between $\mathrm{S}$ and $\mathrm{T}$, and Euclidean distance is used in this paper.

The Hausdorff distance is a measurement which describes the degree of similarity of two sets. $h(S, T)$ describes the similarity from $\mathrm{S}$ to $\mathrm{T}$, and $\mathrm{h}(\mathrm{T}, \mathrm{S})$ exactly the opposite. 
The Hausdorff distance is vulnerable to the noise. Due to the existence of disturbance in character sub-image, the one-way Hausdorff distance means the distance from affine image to character sub-image, which describes the similarity from affine image to character sub-image.

\section{Experiment and Result Analysis}

70244 Chinese characters in National Standards GB180302005 character set are taken as the experimental object. Through splitting each character, summing up all basic elements, some of the basic elements are illustrated in Figure 4. Basic elements and Chinese characters are chosen in 24 pounds Song Ti font and are manufactured in gray images with the size of $32 * 32$ pixels.
Experiment is implemented following the flow persented in Figure 3. Basic elements and Chinese characters are manufactured with the size of $128 * 128$ pixels during the acquisition of the affine coefficients. A demonstration of acquiring the affine coefficients that transforms the basic element image “隹” to the Chinese character image “瞿”, is shown in Figure 5.

SIFT algorithm can produce abundant information of features. There are some mismatched point pairs which are determined by the nearest distance of eigenvectors. One-way Hausdorff distance is used as the similarity measurement between affine image and character subimage in this paper, and three pairs of correct matching points can be identified effectively.

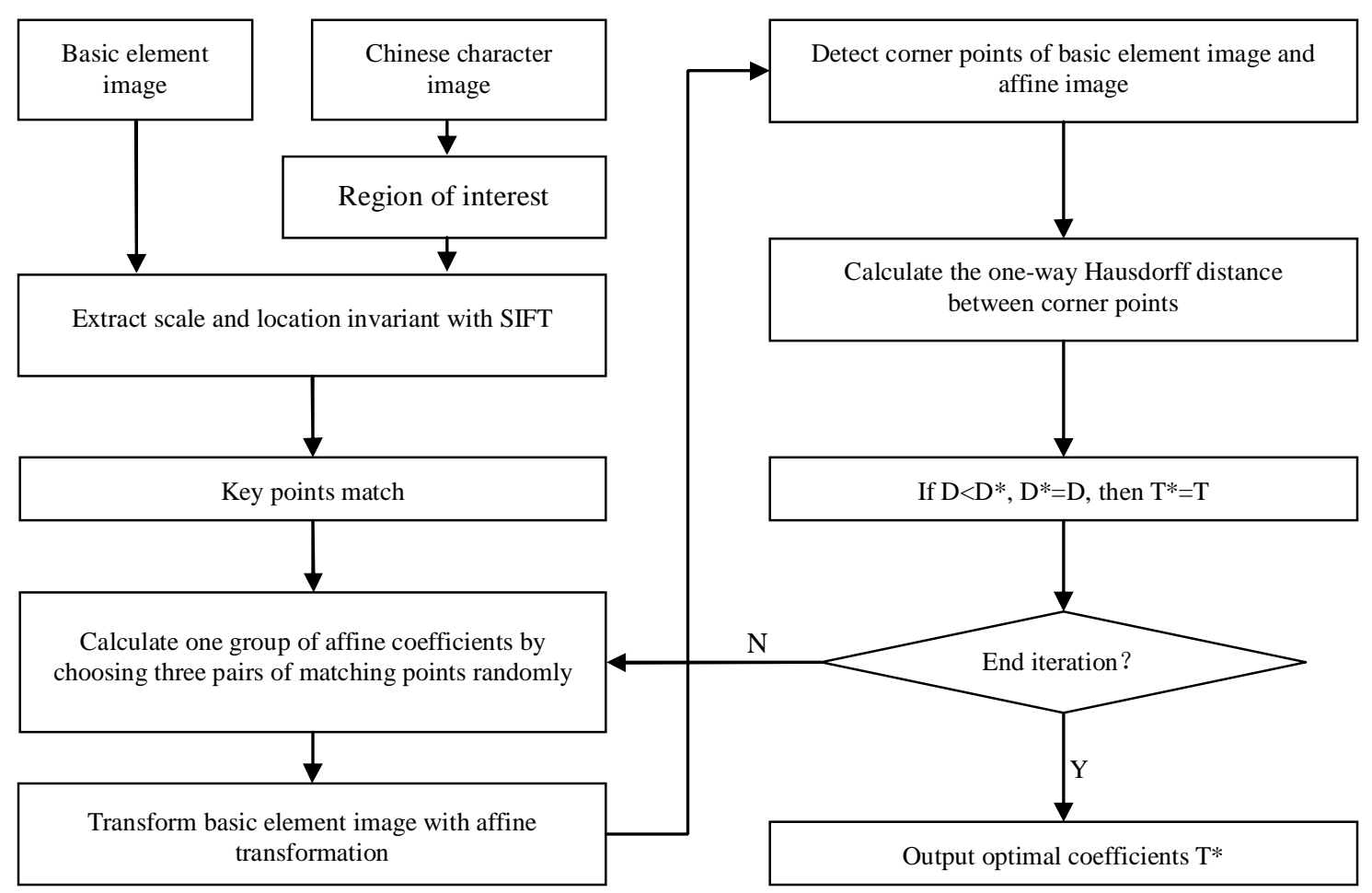

Figure 3. The flow of acquiring affine coefficients

\section{厶尔口多山弓犭皮矢天革田臼 亚赤册元骨用戊文册韦丸《文方妇 疌見应正电差走发爾丰包些兔书}

Figure 4. Some of the basic elements 


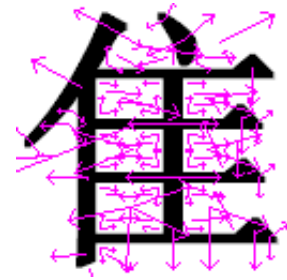

(a)

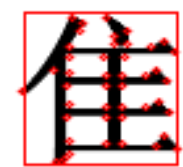

(c)

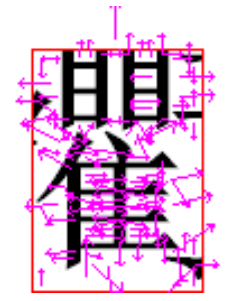

(b)

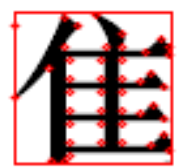

(d)

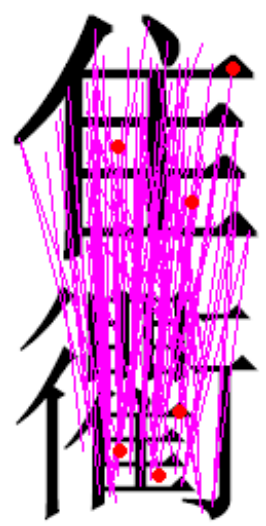

(e)
Figure 5. Example of acquiring affine coefficients. (a) is the basic element image that adds the SIFT features, (b) is Chinese character image that adds the SIFT features, the rectangular box for the interested region, (c) marks one of the affine image's corner points, the rectangular box for the boundary box of affine image, (d) marks the corner points of character sub-image that is corresponding to the rectangular box of (c), (e) is the images that find the optimal three pairs of matching points according to minimum one-way Hausdorff distance

\section{㩫譃橋㴮㵩漂爻㺈浊}

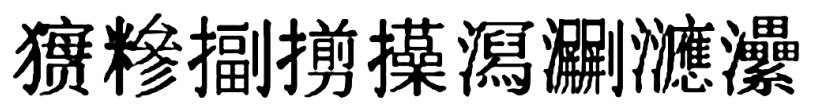
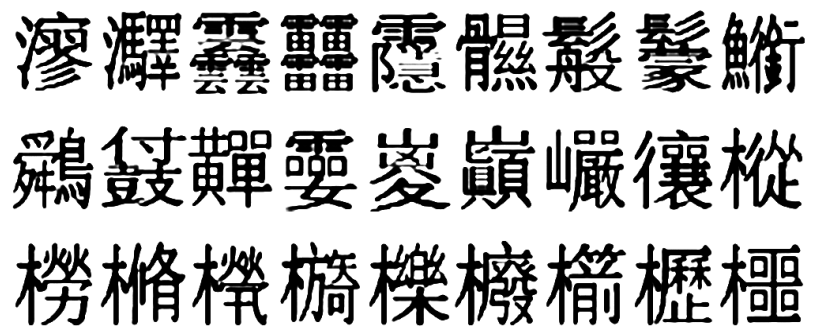

Figure 6. Some of the formed characters
After obtaining the mapping knowledge of all the basic elements, the affine transformations are performed on the basic elements which composed the Chinese characters, then the characters are generated by bitwise AND of the images after the transformation, some of the formed characters are given in Figure 6.

\section{Conclusions}

In this paper, considering the characteristics of the Chinese character, through the extraction of SIFT features, the matching point pairs are determined under the principle of one-way Hausdorff distance, and the affine coefficients are acquired by solving the linear equations. 70244 Chinese characters in the National Standards GB180302005 character set are studied; all the characters are formed successfully. The experimental results demonstrate that the performance of forming characters by basic elements is feasible; the transformation from basic elements to Chinese characters can be realized by affine transformation, and the algorithm that acquires the affine coefficients of basic elements proposed in this paper is efficient. Although the Chinese character study is limited on the size and font of Song Ti in this paper, the proposed method can be extended to other different sizes and fonts.

\section{Acknowledgements}

The author is grateful for the helpful discussion with Jian Huang and Wenzhi Liao.

\section{REFERENCES}

[1] Y. G. Pi, "The formation of chinese character in computerization," CN1558314, China, 2004.

[2] Y. G. Pi, H. L. Shu and T. C. Liang, "The frame of cognitive pattern recognition," In Proceedings of the 26th Chinese Control Conference, Beijing, pp. 694-696, 2007.

[3] T. C. Liang, Z. W. Qiu and Y. G. Pi, "Simple grid based on cognitive mechanism and application research on description for structure of Chinese character," In Proceedings of the 26th Chinese Control Conference, BeiJing, pp, 689-693, 2007.

[4] T. C. Liang and Y. G. Pi, "On Chinese Character Structure(CCS) recognition based on bayesian learning," In Proceedings of the 2007 International Conference on Intelligent Systems and Knowledge Engineering, ChengDu, pp. 1032-1037, 2007.

[5] Y. G. Pi and Z. B. Mou, "The grid and its description of Chinese character in computer," CN1558339, China, 2004.

[6] L. W. Jin and X. N. Zu, "Synthesis of Chinese character using affine transformation," In Proceedings of Ninth International Conference on Document Analysis and Recognition, pp. 218-222, 2007. 
[7] D. G. Lowe, "Object recognition from local scaleinvariant features," In Proceedings of international conference on computer vision, Corfu, Greece, pp. 1150-1157, 1999.

[8] D. G. Lowe, "Distinctive image features from scaleinvariant key-points," International Journal of Computer Vision, Vol. 60,No. 2, pp. 91-110, 2004.
[9] J. B. Shi and C. Tomasi, "Good featrues to track," IEEE Conference on Computer Visoion and Pattern Recognition, Seattle, pp. 593-600, 1994.

[10] B. F. Guo, K. M. Lan and K. H. Lin, " Human face recognition based on spatially weighted Hausdorff distance," Pattern Recognition Letters, Vol. 24, No. 1-3, pp. 499-507, 2003. 